\title{
(2) OPEN ACCESS \\ Spoonful of honey or a gallon of vinegar? A conditional COVID-19 vaccination policy for front-line healthcare workers
}

\author{
Owen M Bradfield ๑ , ${ }^{1}$ Alberto Giubilini $\overbrace{}^{2,3}$
}

\begin{abstract}
${ }^{1}$ Melbourne School of Population and Global Health, The University of Melbourne, Melbourne, Victoria, Australia ${ }^{2}$ Oxford Uehiro Centre for Practical Ethics \& Wellcome Centre for Ethics and the Humanities, University of Oxford, Oxford, UK ${ }^{3}$ University of Oxford, Oxford, UK
\end{abstract}

\section{Correspondence to} Dr Owen M Bradfield Melbourne School of Population and Global Health, The University of Melbourne, Melbourne, VIC 3010, Australia; o.bradfield@student.unimelb. edu.au

Received 17 December 2020 Revised 27 March 2021 Accepted 30 March 2021 Published Online First 11 May 2021

\section{ABSTRACT}

Seven COVID-19 vaccines are now being distributed and administered around the world (figure correct at the time of submission), with more on the horizon. It is widely accepted that healthcare workers should have high priority. However, questions have been raised about what we ought to do if members of priority groups refuse vaccination. Using the case of influenza vaccination as a comparison, we know that coercive approaches to vaccination uptake effectively increase vaccination rates among healthcare workers and reduce patient morbidity if properly implemented. Using the principle of least restrictive alternative, we have developed an intervention ladder for COVID-19 vaccination policies among healthcare workers. We argue that healthcare workers refusing vaccination without a medical reason should be temporarily redeployed and, if their refusal persists after the redeployment period, eventually suspended, in order to reduce the risk to their colleagues and patients. This 'conditional' policy is a compromise between entirely voluntary or entirely mandatory policies for healthcare workers, and is consistent with healthcare workers' established professional, legal and ethical obligations to their patients and to society at large.

\section{INTRODUCTION}

Currently, seven COVID-19 vaccines are being rolled out globally. Six hundred and forty-nine million doses have been administered globally, at a rate of 16.5 million doses per day (figures correct at the time of submission). ${ }^{1}$ As demand initially outweighs supply, vulnerable groups and front-line healthcare workers (FHCWs) are receiving priority access. $^{2}$ FHCWs include healthcare staff who interact directly with patients, including doctors, nurses, allied health clinicians, pathology staff, security, cleaners and students. FHCWs provide essential services in settings that create a high risk of infection and transmission. ${ }^{3}$ Thus, an important ethical question is whether vaccination of FHCWs should be mandatory, as is already the case for influenza immunisation in some US jurisdictions and healthcare facilities. If so, how should this be enforced, and how should the personal preferences and autonomy of FHCWs be balanced against their professional responsibilities to act in the interests of patients and the public? This paper will define and defend a mildly coercive 'conditional' vaccination policy for FHCWs that represents a middle ground between an entirely voluntary and entirely mandatory approach. In our view, this strikes the best balance between the various ethical principles at stake.

\section{JUSTIFYING MANDATORY VACCINATION}

Most public health interventions entail a trade-off between public benefits and private interests. Measures to promote public health and safety invariably create costs, which may be individual (infringement of autonomy), collective (social or economic costs) or often both. In order to justify costs incurred, coercive public health measures should aim to regulate serious risks, not merely 'speculative, theoretical or remote' risks. ${ }^{4}$ In this paper, we adopt Wertheimer's definition according to which 'coercion' involves the threat of penalty for non-compliance and a degree of infringement of individual autonomy that leaves the individual with 'no reasonable choice' or 'no reasonable alternative. ${ }^{5}$ Hence, for the purpose of this paper, a 'mandatory' vaccination policy is considered coercive to the extent that the choice not to vaccinate is rendered difficult or is practically unavailable by the imposition of penalties. Such a policy may take various forms, as outlined below.

The COVID-19 pandemic poses a serious public health threat, as measured by mortality rate, incidence and prevalence. In just over a year, it infected over 131 million people and caused more than 2.8 million deaths. ${ }^{6}$ It disproportionately affects older people. Rates of hospitalisation and death are less than $0.1 \%$ in young children, but increase to more than $10 \%$ in people aged over 70 years. It also disproportionately affects racial and ethnic minorities in terms of frequency and severity of infection. ${ }^{8}$ In healthcare settings, the risks and impacts of COVID-19 are substantial. ${ }^{9}$ FHCWs are at high risk of infection and death, ${ }^{10}$ particularly older FHCWs and those who belong to racial and ethnic minority groups. ${ }^{11}$ FHCWs can transmit the virus to their patients, colleagues, families and the wider community. ${ }^{12}{ }^{13}$ Moreover, hospital patients are generally sicker, ${ }^{14}$ which increases their risk of serious sequelae or death from COVID-19..$^{15}$ When people avoid attending healthcare services for fear of contracting COVID-19, morbidity and mortality from other medical conditions, such as cancer, can increase. ${ }^{16}$ Therefore, there is a pressing need to reduce COVID-19 transmission specifically in healthcare settings and to ensure that patients and FHCWs, particularly those most at risk, do not continue to be disproportionately impacted by COVID-19. Any policy that increases vaccination rates among FHCWs is likely to prevent infection, morbidity and mortality, but needs to ensure equitable access and administration to FHCWs at greatest risk, such as older FHCWs and those from racial and ethnic minority backgrounds.
To cite: Bradfield OM, Giubilini A. J Med Ethics 2021:47:467-472. 
Even if a public health risk is serious, interventions aimed at reducing or preventing those risks must be shown to be effective before their implementation can be morally justified. However, with data yet to prove the effectiveness of a COVID-19 vaccine in healthcare settings specifically, we need to look to analogous situations for guidance. Studies demonstrate that influenzarelated illnesses ${ }^{17}$ and deaths ${ }^{18}$ among elderly inpatients can be significantly reduced when just half of FHCWs are vaccinated against influenza. Mandatory influenza vaccination has been shown to be the most clinically effective ${ }^{19}$ and cost-effective ${ }^{20}$ strategy for increasing vaccination rates among FHCWs. Given the greater threat posed by COVID-19 than influenza in terms of infectivity and mortality, a mandatory COVID-19 vaccination policy could result in substantial benefits in healthcare settings. In fact, early data suggest that widespread and effective vaccination among FHCWs provides a safe hospital working environment, even in the presence of a high rate of SARS-CoV-2 infection in the community. ${ }^{21}$

The benefits of an effective mandatory COVID-19 vaccination policy for FHCWs must be balanced against the cost to FHCWs. No vaccine is $100 \%$ safe. Assuming that any approved COVID-19 vaccine turns out to be no less safe than existing vaccines in routine use, most adverse reactions would likely be relatively minor, except for exceedingly rare complications like vasculitis, encephalomyelitis, neuritis and paralysis (GuillainBarré syndrome). ${ }^{22}$ However, some FHCWs may be at risk of severe adverse vaccine reactions. For instance, the UK government previously advised that FHCWs with a significant history of allergic reactions defer receiving the Pfizer-BioNTech COVID-19 vaccine after two FHCWs reported adverse reactions. ${ }^{23}$

Another cost of mandatory vaccination is that it might undermine goodwill between FHCWs and their employers. Overly coercive regulation may engender resentment, ${ }^{24}$ opposition $^{25}$ and mistrust. FHCWs already experience higher rates of depression, anxiety, insomnia, post-traumatic stress disorder and burn-out from dealing with the tragic reality of this pandemic. ${ }^{26}$ Any mandate needs to be implemented in a way that is respectful and cognisant of the impact that the pandemic is having on the overwhelming majority of FHCWs, most of whom would likely consent to COVID-19 vaccination.

A mandatory vaccination policy also needs to address the cultural and informational needs of diverse racial and ethnic minority groups that have been disproportionately impacted by COVID-19, ${ }^{27}$ particularly since they are also over-represented in front-line healthcare work $^{28}$ and would disproportionately bear the brunt of any vaccine mandate. Adequate resources need to be invested in information and trust campaigns that engage with affected communities and that work with specific cultural or religious views and concerns. To minimise any burdens associated with mandatory vaccination, all FHCWs must be provided with timely, accurate, comprehensible, culturally sensitive and balanced information about the benefits and risks, including areas of uncertainty. In addition, the vaccine must be accessible, offered at very little or no cost and accompanied by robust vaccine injury compensation mechanisms that cover medical expenses arising from vaccine complications. ${ }^{29}$

In most situations, the collective societal benefits of mandatory vaccination will outweigh these small risks of harm. However, individuals should not be required to accept serious existential risks for the sake of public safety. From an ethical perspective, what matters is that the collective benefits outweigh the risks, and that there is some limit to the kind of risks that can be imposed for the sake of those collective benefits. Even if a great collective benefit could be achieved, many would not consider it permissible to impose coercive measures that entail very large individual costs. ${ }^{30}$ For example, if an FHCW has a medical condition that significantly increases the likelihood of a serious adverse vaccine reaction, then it is morally permissible to exempt them from mandatory vaccination policies.

As Flanigan points out, it is permissible for an individual to expose others to risks of harm in order to defend oneself. ${ }^{31}$ By analogy, it may be permissible for an individual to expose others to risks of harm from infectious disease in order to defend oneself from serious vaccine reactions. However, the right to 'self-defence' is not absolute and whether or not an exemption on medical grounds is permitted will depend on the seriousness of the risk of harm to the FHCW. It is difficult to be prescriptive about the type of medical conditions that might authorise a medical exemption until we know more about the immediate and longer term effects of approved COVID-19 vaccines. Giubilini and Savulescu argue that the justification for coercive public health policies is stronger when individuals have a preexisting moral obligation to fulfil those requirements and when such requirements are not overly demanding. ${ }^{32}$ Using other vaccines as an example, we believe that there are compelling ethical reasons to allow medical exemptions within a mandatory COVID-19 vaccination framework.

In view of the risks of vaccination, we need to explore alternatives. Without a vaccine, the use of hand hygiene, personal protective equipment (including face masks, eye goggles, protective suits and face shields), physical distancing, regular surveillance testing and quarantining are our best infection control strategies. In most hospitals, these procedures are already mandatory and FHCWs found to breach infection control guidelines face sanctions, such as disciplinary action or termination. Therefore, while mandatory vaccination may be more burdensome than hand washing, it may be no more burdensome than wearing full personal protective equipment for 12 hours/day. Prolonged use of personal protective equipment can cause physical problems, including breathing difficulties, pain, discomfort and dermatological reactions. ${ }^{33}$ Reducing face-toface clinical contact through telehealth services can also reduce COVID-19 transmission. ${ }^{34}$ However, telehealth services cannot safely and effectively replace all face-to-face healthcare. ${ }^{35}$ So, while other measures exist to control the spread of COVID-19, they are likely inferior to vaccination and not without their own problems. They are, at best, adjuncts to an effective vaccine, but not a replacement. While some countries have achieved shortterm elimination, ${ }^{36}$ this seems an unlikely long-term global goal, with many experts suggesting that COVID-19 will likely become endemic like seasonable influenza. ${ }^{37}$

Hence, assuming that, as is the case with influenza vaccination, mandatory policies are the most effective way of ensuring the highest vaccine uptake possible among FHCWs, mandatory COVID-19 vaccination of FHCWs appears morally justifiable at least from a utilitarian perspective, because it minimises risks and maximises patients' and FHCWs' welfare. The threat posed by COVID-19 is serious, the alternatives available are not a replacement for vaccination, less restrictive policies entail the risk of lower uptake and the public health benefits seem to outweigh the individual risks, except in limited cases where FHCWs are eligible for medical exemptions.

\section{A MILD FORM OF MANDATORY VACCINATION}

Powers that promote public health may permissibly supersede individual liberty. ${ }^{38}$ For example, policies that maximise the number of vaccinated FHCWs promote public health by 
reducing COVID-19 transmission but deprive FHCWs of free choice and autonomy. The principle of the least restrictive alternative may balance these competing interests. ${ }^{39}$ It states that if two public health interventions both effectively address a pressing public health issue, then the least restrictive intervention should be preferred. ${ }^{40}$ The Nuffield Council on Bioethics has articulated an 'intervention ladder ${ }^{41}$ that grades public health policies according to the degree to which they restrict individual autonomy. Less intrusive measures must first be shown to be ineffective before more intrusive measures can be considered. In addition, more restrictive policies will generally achieve greater utility. ${ }^{42}$

At the bottom of the ladder, the least restrictive measure is, of course, no intervention. As explained, this would be unacceptable because vaccination prevents serious risks to patients in healthcare facilities. Persuasion and nudging (in the form of opt-out systems) represent the next rungs up in the ladder, as they seek to influence behaviour and attitudes without depriving individuals of free choice and, according to some accounts, do not compromise autonomy (although this is debated with regard to nudging). Further up the ladder, incentives and disincentives are considered more coercive because they provide people with stronger reasons to do something that they might otherwise prefer not to do. Incentives would likely exert some influence on individuals' decision-making, but it is at least debatable that they are coercive and, on some plausible accounts, they are not. ${ }^{43}$ Disincentives are more clearly coercive and the degree of coercion increases with the size of the disincentive. At the top of the ladder, penalties and force are the most coercive interventions because they leave individuals with few options and limited, if any, free choice. This type of intervention ladder has been applied to vaccination policies for the general population, ${ }^{44}$ but not specifically for FHCWs. So, in table 1, we propose an intervention ladder for vaccination policies for FHCWs.

According to this approach, despite the seriousness of COVID19, coercive vaccination policies would only be required if FHCWs refuse vaccination and persuasion or nudging is ineffective. Given the need for immunity among FHCWs to be achieved rapidly once a vaccine is released, if we adopt this approach healthcare services need to start planning and communicating their proposed vaccination policies early, so that FHCWs objecting to vaccination can be targeted with education campaigns and given advance notice of the consequences of vaccine refusal. Racial, ethnic and other minority groups must receive messaging that addresses particular cultural or religious concerns. In spite of this, persuasion and nudging may be ineffective if the goal is to rapidly achieve close to $100 \%$ vaccination rates. In the case of influenza vaccination, it has been shown that policies requiring declinature statements are far less effective in increasing influenza vaccination rates in institutions aiming to achieve very high vaccination rates. ${ }^{45}$

The principle of least restrictive alternative, although very intuitive, is not without shortcomings. The most obvious is that it does not address the issue of probability of success and of risks. Less restrictive policies are preferable to more restrictive ones if they are both successful. However, if less restrictive policies are less likely to be successful (as is the case with influenza vaccination policies for FHCWs), then it means that by applying the principle we are assuming risk. If the success of the policy in question can prevent great harm-as seems to be the case for COVID-19 vaccination policies-then the risk of failing to achieve or delaying achieving the desired goal might be not worth taking.

If we want to abide by a principle of least restrictive alternative but, at the same time, minimise the risks of lower uptake and of infections in healthcare settings, we could consider a 'conditional' mandate in the first instance that preserves some liberty to refuse vaccination. Contact between unvaccinated FHCWs and vulnerable patients and colleagues could be restricted by redeploying FHCWs to non-clinical administrative duties or telehealth services, assuming their role can be fulfilled by other FHCWs who are vaccinated without significant costs to colleagues and to healthcare systems. This is less restrictive than exclusion from full employment or professional activity.

If redeployment to other roles is not possible, then unvaccinated FHCWs can be asked to take paid or unpaid leave. If, after that period of leave, the FHCW has not been vaccinated, then their employment or professional registration could be suspended or cancelled. Admittedly, this solution could impose significant financial costs on public health services. It could also entail significant professional burdens on vaccinated FHCWs who would be required to assume their unvaccinated colleagues' clinical duties. The question then becomes whether, and to what extent, it is acceptable to impose these collective costs in order to accommodate FHCWs' opposition to vaccines. Some small costs might be a reasonable price to pay, as long as safe and effective healthcare delivery is not compromised. However, it is important to bear in mind that the primary aim of healthcare

Table 1 'Intervention ladder' adapted for mandatory vaccination of FHCWs from most to least coercive

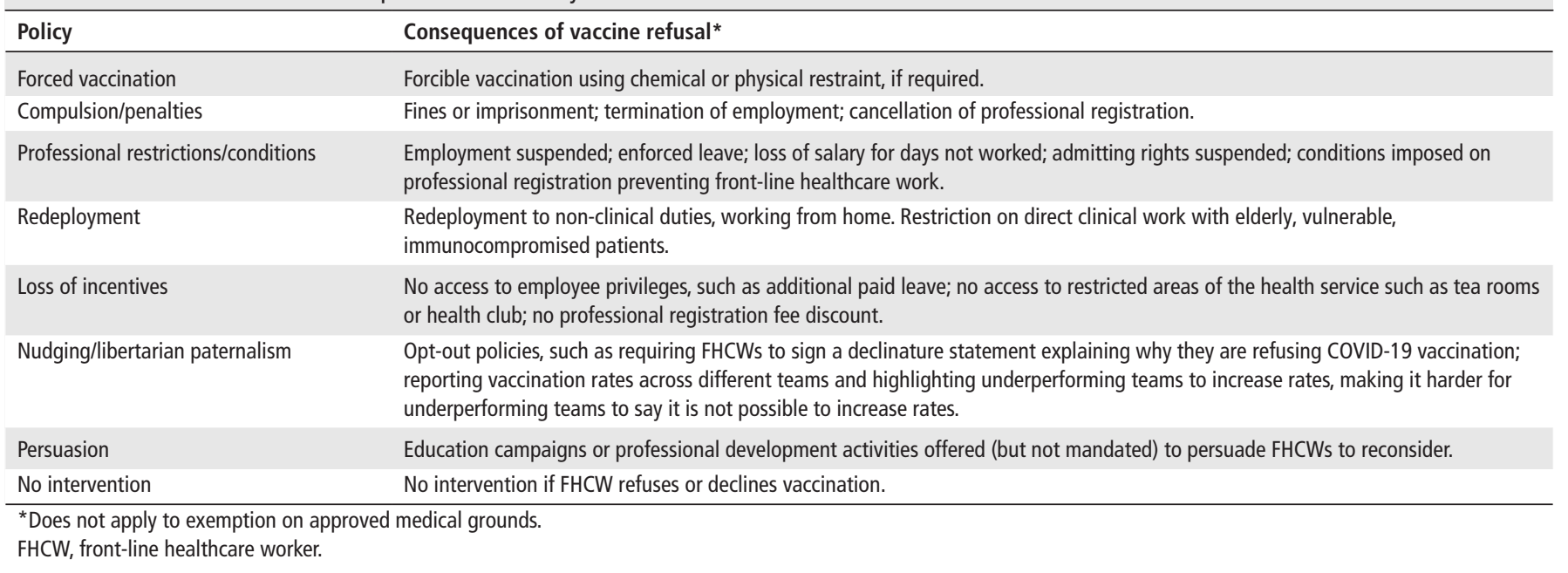


systems and healthcare professions is providing adequate care to patients, and healthcare professionals' personal freedom may and should be constrained accordingly.

Another possible concern with an overly restrictive policy is that it might result in significant workforce understaffing if too many FHCWs refuse vaccination. However, we consider this to be unlikely. Large health service organisations of between 5000 and 40000 staff that adopt mandatory influenza vaccination policies have reported suspending or terminating the employment of no more than eight FHCWs. ${ }^{46}$ Moreover, healthcare services have a general duty to protect the occupational health of their staff. ${ }^{47}$ COVID-19 vaccines might entail more risks than influenza vaccines, but to assess whether it is justifiable to impose such risk on FHCWs through coercive public health policies, we should rely on objective standards of risk assessment, not on FHCWs' subjective perceptions of risk. ${ }^{29}$ Thus, if the risk posed by COVID-19 vaccines is deemed acceptable by some objective standard (such as the one adopted by the authorities that license vaccines), then the risks of vaccination do not defeat mandatory measures. If, instead, the risk is considered high, then mandatory measures might have to be replaced by incentive schemes or supplemented by compensation-for-risk policies, which is a general point that has been made with regard to other forms of risk taking by FHCWs during the pandemic. ${ }^{48}$

Many health services already enforce infection control policies. FHCWs found breaching hand hygiene policies may be disciplined, suspended or reported to their professional registration board. Boards may deem such behaviour, if proven, to constitute unprofessional conduct and may reprimand or suspend an FHCW's registration. Many health services already mandate influenza vaccination for FHCWs. Failure to be vaccinated can result in suspension or even termination of employment. Such action reduces vaccine refusal among FHCWs and improves patient safety. ${ }^{49} \mathrm{FHCW}$ s wo refuse vaccination, other than for genuine and compelling medical reasons, already face the choice of 'no jab, no work'. This might involve annual vaccination certification as a requirement for continued employment, which could be monitored in the same way as other workplace vaccinations and infection control practices. This type of 'fitnessfor-duty' policy already exists in some US hospitals. ${ }^{50}$

Julian Savulescu recently argued that in the case of a novel vaccine about which we have limited long-term safety data, the most appropriate way to incentivise community-wide vaccination might be to compensate individuals for the risk they incur. ${ }^{51}$ $\mathrm{He}$ argues that such a policy avoids coercion and increases peoples' choices. In healthcare settings, incentives might include free meals, professional registration, parking and bonuses. However, unless these incentives are offered to all FHCWs, they may paradoxically discourage many FHCWs from voluntary vaccination if they see colleagues who refused vaccination receiving financial benefits. In addition, incentives may be costly and may be ineffective in persuading those with strong religious or moral convictions against vaccination.

\section{DEFENDING CONDITIONAL VACCINATION}

We have argued for a conditional COVID-19 vaccination policy of FHCWs. One possible objection is that FHCWs' own judgements, moral integrity or personal freedom ought to be prioritised. For instance, some FHCWs may be sceptical about the evidence of safety or effectiveness for a novel vaccine and may prefer to delay or defer vaccination. Some may be opposed on religious grounds. Libertarians might argue that individuals have the right to refuse acts that contravene their beliefs. The right of competent adults to refuse medical treatment, including vaccination, is a well-established ethical principle and is vigorously protected in most common law jurisdictions.

Moral integrity and authenticity require individuals to be faithful to their beliefs. When an individual acts against those beliefs, their identity may be injured. After all, the debate on conscientious objection in healthcare revolves precisely around this point. FHCWs may conscientiously object to providing contraception, pregnancy terminations or assistance with voluntary dying. ${ }^{5253}$ In the same way, they may conscientiously object to vaccination. ${ }^{54}$ In fact, FHCWs refusing vaccination might argue that, unlike conscientious objection to abortion or assisted dying, they are refusing to treat themselves instead of refusing to treat their patients. In fact, it has even been argued that the right to conscientious objection in healthcare protects individuals and society ${ }^{55}$ because FHCWs committed to upholding moral ideals are resisting moral compromises that might otherwise lead to unethical or unprofessional conduct. ${ }^{56}$ However, leaving aside the complexities of the debate around conscientious objection in healthcare, these arguments as applied to vaccine refusal among FHCWs fail for a number of reasons.

First, the right to exercise free choice is not absolute. According to John Stuart Mill, personal freedoms extend only so far as they do not infringe on the legitimate interests of others. ${ }^{57}$ The European Convention on Human Rights also recognises that freedom of thought, conscience and religion may be limited by public safety concerns. ${ }^{58}$ The British Medical Association's guidance on conscientious objection, for instance, recognises that, where a conflict arises between the interests of a patient and a doctor's freedom to conscientiously object, the conflict should be resolved in favour of the patient. ${ }^{59}$ This is consistent with our argument.

Second, and more critically, FHCWs owe a professional and ethical obligation of non-maleficence towards their patients. One of the foundational principles of healthcare ethics is to 'First Do No Harm ${ }^{6}{ }^{60}$ Failing to avoid infecting others is ethically tantamount to harm through omission. ${ }^{61}$ Vaccine refusal puts patients at risk of infection and death. Given the evidence that vaccination prevents disease transmission to vulnerable patients and maintains the health of FHCWs, vaccination should be seen as a fundamental moral requirement for all FHCWs. The duty not to infect patients must take priority over any right to vaccine refusal. Indeed, most defenders of conscientious objection in healthcare concede that the duty to guarantee patients' access to healthcare takes priority over FHCWs' conscientious objection to practices like abortion. ${ }^{62}$ In our view, the same applies to vaccination.

Consider a case in which one individual risks sexually transmitting HIV to another individual by not wearing a condom. There is of course no obligation not to contract COVID-19 (or HIV), though there might be an obligation to minimise the risk of contracting it. In both cases, there is an unequivocal duty not to infect another person when this can be easily prevented, either by using condoms or being vaccinated. Vaccination is sufficiently easy and costless for most FHCWs that a positive duty to be vaccinated against COVID-19 could be as compelling as negative duties to avoid infecting patients with COVID-19. Since vaccination entails a very small cost to most FHCWs and a very large benefit to patients, FHCWs owe their patients a duty of care or even a duty of easy rescue $e^{63}$ to be vaccinated.

Third, promoting the autonomy of FHCWs should not confine the autonomy of patients. Few patients can choose their FHCW. While mandatory vaccination policies are coercive for FHCWs, not instituting mandatory vaccination may be more 
coercive for patients. Whereas FHCWs can choose between vaccination and their job, patients cannot choose whether or not to get sick and cannot choose who cares for them when sick. They might have no alternative but be treated and cared for by unvaccinated FHCW. Therefore, the restriction on choice experienced by sick patients if no mandatory vaccination is imposed on FHCWs could be greater than the restriction experienced by FHCWs if such a policy is imposed.

Fourth, mandatory vaccination is arguably less burdensome than other mandatory infection control practices, as previously described. Employers and medical regulators already mandate infection control procedures on FHCWs that are restrictive and cause difficulty breathing, physical pain and skin breakdown. In addition, many health practitioner licensing boards already require FHCWs to be regularly tested for bloodborne viral infections, in recognition of their professional responsibility to prevent transmission to their patients. ${ }^{64}$ Requiring FHCWs to be vaccinated against COVID-19 is entirely consistent with established professional codes of conduct and ethics in many jurisdictions.

Fifth, there are concerns that the COVID-19 pandemic has revived antivaccine sentiments. ${ }^{65}$ Surveys show that public willingness to be vaccinated against COVID-19 varies from $49 \%$ in the USA ${ }^{66} 62 \%$ in France, $80 \%$ in Denmark, ${ }^{67} 85 \%$ in Australia, ${ }^{68}$ to $93 \%$ in Indonesia. ${ }^{69}$ Willingness also varies according to race and ethnicity. ${ }^{70}$ The right of FHCWs to act on their personal views about COVID-19 vaccines must be balanced against the adverse consequences of their personal decisions on public health messaging. Hence, another powerful reason to mandate COVID-19 vaccination of FHCWs is to dispel public mistrust of vaccination and to encourage FHCWs to lead by example. Public trust in the healthcare system might diminish if it became known that FHCWs are forgoing vaccination and failing to prevent their patients getting sick or dying. ${ }^{71}$

Finally, every effort should be made to ensure that public health messaging about the benefits and risks of COVID-19 vaccination reaches disadvantaged groups, especially FHCWs from racial and ethnic minority groups that are over-represented in front-line healthcare roles and disproportionately impacted by serious adverse COVID-19 health outcomes. This benefits racially and ethnically diverse FHCWs themselves, and it assists them to spread important public health messages within their own culturally and linguistically diverse communities to ensure that coercive vaccination policies do not unfairly target and further disadvantage already marginalised groups.

\section{CONCLUSION}

We need to consider how COVID-19 vaccines should be ethically distributed and what to do if people refuse to be vaccinated, given the impact that individual vaccine decisions have on society and specific communities. Within healthcare settings, vaccine choices can have even greater ramifications which, when coupled with the seriousness of COVID-19, justifies at least a mild form of mandatory vaccination policy for FHCWs. We believe that this should take the form of conditional employment or conditional professional registration, although temporary redeployment could be adopted if this does not entail significant costs to patients, to vaccinated colleagues and to the healthcare system. It is plausible that this would be the least restrictive policy that is most likely to achieve adequate vaccination uptake to reduce transmission of COVID-19 from unvaccinated FHCWs to their patients and colleagues and satisfy ethical and professional requirements. The risk to patients and the community posed by unvaccinated FHCWs outweighs concerns that conditional vaccination policies are coercive, provided that public health messages engage with people from diverse communities and groups within society.

Acknowledgements The authors acknowledge Professor Dominic Wilkinson, who provided helpful comments on an early draft of this paper.

Contributors $\mathrm{OMB}$ developed the concept for the article with consultation and expansion from AG. OMB performed the scoping and primary drafting while AG provided substantial revisions and expansion of concepts. Both OMB and AG approved the final version of the manuscript.

Funding OMB was supported by a Melbourne Research Scholarship and is also a recipient of a 2020 Fulbright Future Scholarship sponsored by The Kinghorn Foundation. AG was supported by an AHRC/UKRI grant (AH/V006819/1).

Competing interests None declared.

Patient consent for publication Not required.

Provenance and peer review Not commissioned; externally peer reviewed.

Data availability statement There are no data in this work.

Open access This is an open access article distributed in accordance with the Creative Commons Attribution 4.0 Unported (CC BY 4.0) license, which permits others to copy, redistribute, remix, transform and build upon this work for any purpose, provided the original work is properly cited, a link to the licence is given, and indication of whether changes were made. See: https://creativecommons.org/ licenses/by/4.0/.

\section{ORCID iDs}

Owen M Bradfield http://orcid.org/0000-0002-8955-7432

Alberto Giubilini http://orcid.org/0000-0001-5163-3017

\section{REFERENCES}

1 Our World in Data. Coronavirus (COVID-19) vaccination. Available: https:// ourworldindata.org/covid-vaccinations [Accessed 5 April 2021].

2 Frederiksen LSF, Zhang Y, Foged C, et al. The long road toward COVID-19 herd immunity: vaccine platform technologies and mass immunization strategies. Front Immunol 2020;11:1817.

3 Giubilini A, Savulescu J, Wilkinson D. COVID-19 vaccine: vaccinate the young to protect the old? J Law Biosci 2020;7(1):Isaa050.

4 Gostin LO, Wiley LF. Public health law: power, duty, restraint. Berkeley: University of California Press, 2016: 40

5 Wertheimer A. Remarks on coercion and exploitation. Denv UL Rev 1996;74.

6 Worldometer. COVID-19 coronavirus pandemic. Available: https://www.worldometers. info/ coronavirus/ [Accessed 5 April 2021].

7 Mallapaty S. The coronavirus is most deadly if you are older and male - new data reveal the risks. Nature 2020;585(7823):16-17.

8 Azar KMJ, Shen Z, Romanelli RJ, et al. Disparities in outcomes among COVID-19 patients in a large health care system in California. Health Aff 2020;39(7):1253-62.

9 Burton JK, Bayne G, Evans C, et al. Evolution and effects of COVID-19 outbreaks in care homes: a population analysis in 189 care homes in one geographical region of the UK. Lancet Healthy Longev 2020;1(1):e21-31.

10 Kua J, Patel R, Nurmi E, et al. healthcareCOVID: a national cross-sectional observational study identifying risk factors for developing suspected or confirmed COVID-19 in UK healthcare workers. PeerJ 2021;9:e10891.

11 Royal College of Psychiatrists. Impact of COVID-19 on black, Asian and minority ethnic (BAME) staff in mental healthcare settings | assessment and management of risk, 2020. Available: https://www.rcpsych.ac.uk/docs/default-source/about-us/covid19/impact-of-covid19-on-bame-staff-in-mental-healthcare-settings-report-2020.pdf? sfvrsn=22a9083a_2 [Accessed 18 Feb 2021].

12 Nguyen LH, Drew DA, Graham MS, et al. Risk of COVID-19 among front-line healthcare workers and the general community: a prospective cohort study. Lancet Public Health 2020;5(9):e475-83.

13 Hancock J. Coronavirus outbreak at Melbourne's Box Hill Hospital a 'canary in the coalmine', doctor warns. ABC News Online. Available: https://www.abc.net.au/news/ 2020-10-13/melbourne-coronavirus-covid-19-outbreak-box-hill-hospital/12758642 [Accessed 14 Nov 2020].

14 Valderas JM, Starfield B, Sibbald B, et al. Defining comorbidity: implications for understanding health and health services. Ann Fam Med 2009:7(4):357-63.

15 Khan KS, Reed-Embleton H, Lewis J, et al. Does nosocomial COVID-19 result in increased 30-day mortality? A multi-centre observational study to identify risk factors for worse outcomes in patients with COVID-19. J Hosp Infect 2021;107:91-4.

16 Lazzerini M, Barbi E, Apicella A, et al. Delayed access or provision of care in Italy resulting from fear of COVID-19. Lancet Child Adolesc Health 2020;4(5):e10-11.

17 Wang C-S, Wang S-T, Lai C-T, et al. Impact of influenza vaccination on major causespecific mortality. Vaccine 2007;25(7):1196-203.

18 Carman WF, Elder AG, Wallace LA, et al. Effects of influenza vaccination of health-care workers on mortality of elderly people in long-term care: a randomised controlled trial. Lancet 2000;355(9198):93-7. 
19 Lytras T, Kopsachilis F, Mouratidou E, et al. Interventions to increase seasonal influenza vaccine coverage in healthcare workers: a systematic review and meta-regression analysis. Hum Vaccin Immunother 2016;12(3):671-81.

20 Burls A, Jordan R, Barton $\mathrm{P}$, et al. Vaccinating healthcare workers against influenza to protect the vulnerable--is it a good use of healthcare resources? A systematic review of the evidence and an economic evaluation. Vaccine 2006;24(19):4212-21.

21 Benenson S, Oster Y, Cohen MJ, et al. BNT162b2 mRNA Covid-19 vaccine effectiveness among health care workers. N Eng/ J Med 2021. doi:10.1056/ NEJMc2101951. [Epub ahead of print: 23 Mar 2021].

22 van Delden JJM, Ashcroft R, Dawson A, et al. The ethics of mandatory vaccination against influenza for health care workers. Vaccine 2008;26(44):5562-6.

23 Smout A. UK issues anaphylaxis warning on Pfizer vaccine after adverse reactions. Reuters, 2020. Available: https://www.reuters.com/article/health-coronavirus-britainvaccine/uk-warns-people-with-serious-allergies-to-avoid-pfizer-vaccine-after-twoadverse-reactions-idUSKBN28J1D1 [Accessed 15 Dec 2020].

24 Winston L, Wagner S, Chan S. Healthcare workers under a mandated H1N1 vaccination policy with employment termination penalty: a survey to assess employee perception. Vaccine 2014;32(37):4786-90.

25 Ksienski DS. Mandatory seasonal influenza vaccination or masking of British Columbia health care workers: Year 1. Can J Public Health 2014;105(4):e312-6.

26 Lai J, Ma S, Wang Y, et al. Factors associated with mental health outcomes among health care workers exposed to coronavirus disease 2019. JAMA Netw Open 2020;3(3).

27 Tai DBG, Shah A, Doubeni CA, et al. The disproportionate impact of COVID-19 on racial and ethnic minorities in the United States. Clin Infect Dis 2021;72(4):703-6.

28 Platt L, Warwick R. Are some ethnic groups more vulnerable to COVID-19 than others? Institute for Fiscal Studies, 2020. Available: https://www.ifs.org. uk/inequality/wp-content/uploads/ 2020/04/Are-some-ethnic-groups-morevulnerable-to-COVID-19-than-others-V2-IFS-Briefing-Note.pdf [Accessed $25 \mathrm{Mar}$ 2021].

29 Mello MM, Silverman RD, Omer SB. Ensuring uptake of vaccines against SARS-CoV-2. N Engl J Med 2020;383(14):1296-9.

30 Giubilini A, Douglas T, Maslen $\mathrm{H}$, et al. Quarantine, isolation and the duty of easy rescue in public health. Dev World Bioeth 2018:18(2):182-9.

31 Flanigan J. A defense of compulsory vaccination. HEC Forum 2014;26(1):5-25.

32 Giubilini A, Savulescu J. Demandingness and public health ethics. Moral Philos Politics 2019:6(1):65-87.

33 Shaukat N, Ali DM, Razzak J. Physical and mental health impacts of COVID-19 on healthcare workers: a scoping review. Int J Emerg Med 2020;13(1):1-8.

34 Lee I, Kovarik C, Tejasvi T, et al. Telehealth: helping your patients and practice survive and thrive during the COVID-19 crisis with rapid quality implementation. J Am Acad Dermatol 2020:82(5):1213-4.

35 Fagherazzi G, Goetzinger C, Rashid MA, et al. Digital health strategies to fight COVID-19 worldwide: challenges, recommendations, and a call for papers. J Med Internet Res 2020;22(6):e19284.

36 Baker MG, Kvalsvig A, Verrall AJ. New Zealand's COVID-19 elimination strategy. Med Aust 2020;213(198):10-5694.

37 Shaman J, Galanti M. Will SARS-CoV-2 become endemic? Science 2020;370(6516):527-9.

38 Rothstein MA. Rethinking the meaning of public health. J Law Med Ethics 2002:30(2):144-9.

39 Childress JF, Faden RR, Gaare RD, et al. Public health ethics: mapping the terrain. J Law Med Ethics 2002:30(2):170-8.

40 Saghai Y. Radically questioning the principle of the least restrictive alternative: a reply to Nir Eyal: Comment on "Nudging by Shaming, Shaming by Nudging". Int J Health Policy Manag 2014;3(6):349-50.

41 Nuffield Council on Bioethics. Public health: ethical issues, 2007. Available: https:// www. nuffieldbioethics.org/assets/pdfs/Public-health-ethical-issues.pdf [Accessed 20 Nov 2020].

42 Briggs RA. Normative Theories of Rational Choice: Expected Utility. In: Zalta EN, ed The Stanford encyclopedia of philosophy (Fall 2019 Edition), 2019. https://plato. stanford.edu/archives/fall2019/entries/rationality-normative-utility/

43 Grimwade O, Savulescu J, Giubilini A, et al. Payment in challenge studies: ethics, attitudes and a new payment for risk model. J Med Ethics 2020:46(12):815-26.

44 Giubilini A. The ethics of vaccination. Berlin: Springer Nature, 2019
45 Polgreen PM, Septimus EJ, Parry MF, et al. Relationship of influenza vaccination declination statements and influenza vaccination rates for healthcare workers in 22 US hospitals. Infect Control Hosp Epidemiol 2008;29(7):675-7.

46 Babcock HM, Gemeinhart N, Jones M, et al. Mandatory influenza vaccination of health care workers: translating policy to practice. Clin Infect Dis 2010;50(4):459-64.

47 Sinclair RR, Allen T, Barber L, et al. Occupational health science in the time of COVID-19: now more than ever. Occup Health Sci 2020;4(1-2):1-22.

48 Giubilini A, Savulescu J. Stopping exploitation: properly remunerating healthcare workers for risk in COVID-19 pandemic. Bioethics 2020.

49 Kitt $\mathrm{E}$, Burt S, Price SM, et al. Implementation of a mandatory influenza vaccine policy: a 10-year experience. Clin Infect Dis 2020. doi:10.1093/cid/ciaa782. [Epub ahead of print: 17 Jun 2020]

50 Rakita RM, Hagar BA, Crome P, et al. Mandatory influenza vaccination of healthcare workers: a 5-year study. Infect Control Hosp Epidemiol 2010;31(9):881-8.

51 Savulescu J. Good reasons to vaccinate: mandatory or payment for risk? J Med Ethics 2021:47(2):78-85

52 General Medical Council. Personal beliefs and medical practice. Available: https:// www.gmc-uk.org/ethical-guidance/ethical-guidance-for-doctors/personal-beliefs-andmedical-practice/personal-beliefs-and-medical-practice [Accessed 22 Nov 2020].

53 Australian Medical Association. Position statement on conscientious objection, 2019. Available: https://ama.com.au/position-statement/conscientious-objection-2019 [Accessed 22 Nov 2020].

54 Navin MC, Largent MA. Improving nonmedical vaccine exemption policies: three case studies. Public Health Ethics 2017:12(3):225-34.

55 Magelssen M. When should conscientious objection be accepted? J Med Ethics 2012:38(1):18-21.

56 Gerrard JW. Is it ethical for a general practitioner to claim a conscientious objection when asked to refer for abortion? J Med Ethics 2009;35(10):599-602.

57 Feinberg J. Rights, justice, and the bounds of liberty: essays in social philosophy. Princeton University Press, 2014.

58 European convention on human rights;9.

59 British Medical Association. Expressing your personal beliefs as a doctor. Available: https://www.bma.org.uk/advice-and-support/ethics/personal-ethics/expressing-yourpersonal-beliefs-as-a-doctor [Accessed 22 Nov 2020].

60 van den Hoven MA, Verweij MF. Should we promote influenza vaccination of health care workers in nursing homes? some ethical arguments in favour of immunization. Age Ageing 2003:32(5):487-9.

61 Harris J, Holm S. Is there a moral obligation not to infect others? BMJ 1995;311(7014):1215-7.

62 Wicclair MR. Conscientious objection in health care: an ethical analysis. Cambridge University Press, 2011.

63 Singer P. Famine, affluence, and morality. . Philosophy and Public Affairs, 1972: 1. 229-43.

64 Medical Board of Australia. Guidelines: registered health practitioners and students in relation to blood-borne viruses, effective 6 July 2020. Available: https://www.ahpra. gov.au/documents/default.aspx?record=WD20\%2f29836\&dbid=AP\&chksum=bkwP hCQ9D854WSN5V7f0jw\%3d\%3d [Accessed 23 Nov 2020].

65 Hotez PJ. COVID19 meets the antivaccine movement. Microbes Infect 2020;22(4 5):162-4.

66 Associated Press-NORC Center for Public Affairs Research. Expectations for a COVID-19 vaccine, 2020. Available: http://www.apnorc.org/projects/Pages/ Expectations-for-a-COVID-19-Vaccine.aspx

67 Neumann-Böhme S, Varghese NE, Sabat I, et al. Once we have it, will we use it? A European survey on willingness to be vaccinated against COVID-19. Eur J Health Econ 2020;21(7):977-82.

68 Dodd RH, Cvejic E, Bonner C, et al. Willingness to vaccinate against COVID-19 in Australia. Lancet Infect Dis 2021;21(3):318-9.

69 Harapan H, Wagner AL, Yufika A, et al. Acceptance of a COVID-19 vaccine in Southeast Asia: a cross-sectional study in Indonesia. Front Public Health 2020;8.

70 Painter EM, Ussery EN, Patel A, et al. Demographic Characteristics of Persons Vaccinated During the First Month of the COVID-19 Vaccination Program - United States, December 14, 2020-January 14, 2021. MMWR Morb Mortal Wkly Rep 2021;70(5):174-7

71 Poland GA. Mandating influenza vaccination for health care workers: putting patients and professional ethics over personal preference. Vaccine 2010;28(36):5757-9. 